\section{Nuclear Medicine}

\section{SELF-ASSESSMENT QUESTIONNAIRE}

\section{SAQs - and answers - are ONLINE for RCP Fellows and Collegiate Members}

The SAQs printed in the CME section can be answered online to achieve External CPD credits.

\section{The answering process}

1. To access the questions, log on to the Fellows and Members area http://www.rcplondon.ac.uk/Members/SAQ (those who have not yet registered will be automatically directed to the registration pages)

2. Select: Online learning $S A Q$

3. At the top of the SAQ page select the current CME question paper

4. Answer all 10 questions in any order, by indicating true or false

5. Check your answers and change them if you wish to

6. Click on Submit for final marking.

(Note - after submitting your answers NO changes are possible)

\section{The marking process}

- You must submit the answers before the closing date shown at the top of the screen

- Answers will be marked automatically on the date displayed for that paper

- You can find your marks with explanations of the answers on the CME page under My past CME papers

\section{Registering your External CPD credits}

A pass mark of $80 \%$ allows you to claim 2 External CPD credits. Thus by answering the SAQs in each issue of Clinical Medicine you can achieve 12 external credits in one year.

To claim your credits:

- Online registrants: You can record your credits using the online diary system. All Clinical Medicine SAQs are listed under External Approved CPD

- Manual registrants: You can record your credits using your paper diary sheets. Manual registrants are required to keep evidence of their participation in the SAQ and the score attained.

Please note that past papers will be stored for 12 months.

For some time we have run the SAQs in paper and electronic form. From the September/October 2006 issue we will be moving to an on-line only system. If there are any problems with this change, please contact clinicalmedicine@rcplondon.ac.uk or write to the

Publications Department, Royal College of Physicians,

11 St Andrews Place, London NW1 4LE
1 A 44-year-old man with Hodgkin's lymphoma has anterior mediastinal lymphadenopathy but no disease elsewhere. He is treated with a conventional chemotherapy regimen and is clinically well with a normal clinical examination. Which of the following statements are true and which false?

(a) If there are normal size nodes on computed tomography (CT), active disease can be excluded

(b) 18F-fluorodeoxyglucose (FDG)/positron emission tomography (PET) imaging should be used to assess for disease remission

(c) A FDG PET/CT scan performed after only two cycles of chemotherapy accurately predicts future prognosis

(d) Positive FDG uptake in the lungs during or shortly after chemotherapy is highly suggestive of pulmonary lymphoma

(e) If the mediastinal mass shows only partial resolution on CT, negative FDG uptake would be unreliable in confirming remission

2 A 65-year-old man with newly diagnosed sigmoid carcinoma is undergoing pre-operative evaluation. Clinical examination is normal. Which of the following statements are true and which false?

(a) $\mathrm{PET} / \mathrm{CT}$ is indicated in his pre-operative work-up

(b) $\mathrm{PET} / \mathrm{CT}$ is indicated as an alternative to colonoscopy to detect synchronous colonic primaries

(c) Suspected recurrent colorectal tumour following colonic resection should be evaluated with PET/CT

(d) If the patient develops a recurrent colorectal tumour and liver metastases detected on contrast enhanced CT, further imaging with PET imaging is unnecessary

(e) Abnormal FDG uptake may be seen following pelvic radiotherapy

3 A 75-year-old woman suffered a T11 spinal fracture two weeks previously. She had suffered bilateral Colles fractures in her 50s. Which methods are appropriate for diagnosis of osteoporosis in this patient?

(a) Hip dual-energy X-ray absorptiometry (DXA)

(b) Spine and hip DXA

(c) Spine, hip and wrist DXA 
(d) Heel ultrasound

(e) X-ray

4 A $99 \mathrm{~m} T$ Tc-mercaptoacetyltriglycine-3 renogram will assist with the diagnosis or follow-up of the following conditions:

(a) Diagnosis of pelvi-ureteric junction obstruction

(b) Diagnosis of ureterocoele

(c) Diagnosis of acute tubular necrosis in a patient with a renal transplant

(d) Monitoring progression of scarring in a patient with a renal transplant

(e) Monitoring progression and timing of intervention in a patient with a urinary calculus

5 A 42-year-old woman with amenorrhoea for three months presents for DXA screening following a fragility fracture of the wrist. She has a past history of anorexia nervosa in her 20s and Graves' disease which was treated with radioactive iodine following 18 months of carbimazole. She has a three-month history of epigastric pain. The DXA scan should be delayed if:

(a) A barium swallow was performed 24 hours previously

(b) The patient is unwilling to remove a corset with metal stays

(c) The patient underwent a CT with intravenous contrast 24 hours previously

(d) The patient is pregnant

(e) The patient has acute sciatica which prevents her from lying on the scanning bed even for two minutes

6 A 70-year-old woman is seen for assessment for treatment of osteoporosis. She has a T12 fragility fracture. Her bone density $T$ score is -3.0 in the hip and -3.6 in the spine. There is no history of gastrooesophageal reflux. According to the National Institute for Health and Clinical Excellence guidelines, which of the following is appropriate management?
(a) Alendronate
(b) Residronate
(c) Raloxifene
(d) Teriparatide
(e) No treatment

7 A 45-year-old woman with atypical chest pain and severe asthma is referred for myocardial perfusion scintigraphy (MPS).

Unfortunately, she omitted to read her patient information letter and drank several cups of coffee before leaving for her appointment. Which of the following statements are true and which false?

(a) The patient is suitable for adenosine stress

(b) The patient is suitable for exercise stress

(c) The patient is suitable for dobutamine stress

(d) MPS is a useful initial test in women with stable chest pain

(e) Normal MPS indicates a favourable prognosis

8 A 60-year-old man presents with recent onset dyspnoea and anginal type chest pain. $\mathrm{He}$ is referred for MPS which shows severe reversible defects in the anteroseptal region and lateral wall. There is thallium uptake in the lungs on the stress images. Which of the following statements are true and which false?

(a) The patient is at high risk of future coronary events

(b) The left anterior descending coronary artery is likely to be involved

(c) The patient has single-vessel disease

(d) ECG-gated MPS would provide additional information about left ventricular function

(e) The scan indicates that the patient has sustained an extensive infarct

9 A 50-year-old man (height $175 \mathrm{~cm}$, weight $120 \mathrm{~kg}$ ) presents to his local hospital with recurrent angina. He underwent percutaneous intervention to the right coronary artery two months previously. The patient is referred for MPS with the request form stating 'Obesity. Recent percutaneous intervention. Recurrent chest pain'. Which of the following statements are true and which false?

(a) Details of the patient's coronary anatomy and site of percutaneous intervention are irrelevant to the reporting physician

(b) The reference to obesity is sufficient to allow the nuclear cardiology provider to plan a suitable imaging protocol

(c) Normal MPS excludes functionally significant angioplasty re-stenosis

(d) A reversible inferior perfusion defect could be safely assigned to the right coronary artery

(e) A reversible perfusion defect seen two weeks after angioplasty indicates angioplasty re-stenosis

10 A 75-year-old man with widespread bone metastases from prostate cancer receives radionuclide therapy for bone pain palliation. Which of the following statements are true and which false?

(a) The use of ${ }^{89} \mathrm{Sr}$ for bone pain in prostate cancer is curative

(b) Pain relief starts within 12 hours following ${ }^{89} \mathrm{Sr}$ therapy

(c) The response rate for pain relief is $40-50 \%$

(d) I-131 can be used for bone pain palliation

(e) ${ }^{177}$ Lu somatostatin analogues can be used for bone pain palliation 